\title{
Amadeus
}

International Multidisciplinary Journal ISSSN 2525-8281

DOI: 10.14295/aimj.v4i7.93

\section{Epidemiological Determinants of Cranioencephalic Trauma in Children}

Daniel Victor Lima Gonçalves ${ }^{l}$;

Pedro de Sousa Leite ${ }^{2}$;

Bráulio Filgueira Magalhães ${ }^{3}$; Emanuela Fonseca Cruz;

Amanda Fonseca Cruz ;

Cicero Hyttallo Carneiro

Balduino ${ }^{6}$;

Emanuelly Gonçalves Saraiva ${ }^{7}$; Thiago Moreira de Alencar ${ }^{8}$;

Natália Moreira Garcia

Feitosa ${ }^{9}$

Virgínia Moreira Garcia

Feitosa $^{10}$
Abstract: This article aims to evaluate the epidemiological determinants of TBI in pediatric patients. An integrative review was performed by searching indexed articles in the Virtual Health Library, in which analysis after final analysis included 08 articles. For better elucidation, two categories were formulated: Epidemiological profile of children with TBI and Epidemiological determinants and the clinical implications of TBI in children. It was possible to verify that there is a male predominance, the fall is considered the main mechanism for the condition and TBI, in addition, age groups under 5 years have a worse prognosis.

Keywords: Craniocerebral Trauma; Child; Epidemiologic Factors.

\footnotetext{
${ }^{1}$ Médico formado pela Universidade Ceuma - Maranhao. danielimapsi@gmail.com . Juazeiro do Norte, Ceará, Brasil;

${ }^{2}$ Graduando em Medicina da Faculdade de Medicina Estácio de Juazeiro do Norte. Pedroed1913@ hotmail.com , (88) 996736749. Juazeiro do Norte, Ceará, Brasil;

Residente em Cirurgia Pela Faculdade de Faculdade de Medicina Estácio de Juazeiro do Norte. braulio.magalhaes@ hotmail.com .Juazeiro do Norte, Ceará, Brasil;

${ }^{4}$ Médica formado pela Universidade Ceuma - Maranhao. Emanuelafonseca@ yahoo.com.br . Juazeiro do Norte, Ceará, Brasil;

${ }^{5}$ Médica formado pela Universidade Ceuma - Maranhao. Amandafonsecacruz16@gmail.com . Juazeiro do Norte, Ceará, Brasil;

${ }^{6}$ Graduando em Medicina da Faculdade de Medicina Estácio de Juazeiro do Norte. hyttallo@hotmail.com . Juazeiro do Norte, Ceará, Brasil;

7 Graduanda em Medicina da Faculdade de Medicina Estácio de Juazeiro do Norte. emanuellygoncalves@gmail.com . Juazeiro do Norte, Ceará, Brasil;

${ }^{8}$ Médico Formação UCEBOL Bolívia - revalidado pela UFMT. Juazeiro do Norte, Ceará, Brasil;

9 Graduanda em Medicina da Faculdade de Medicina Estácio de Juazeiro do Norte. nataliafeitosa_garcia@hotmail.com . Juazeiro do Norte, Ceará, Brasil;

10 Graduanda em Medicina da Faculdade de Medicina Estácio de Juazeiro do Norte. virginiamgfeitosa@gmail.com . Juazeiro do Norte, Ceará, Brasil;
} 


\section{Determinantes Epidemiológicos do Traumatismo Cranioencefálico em Crianças}

\begin{abstract}
Resumo: O presente artigo tem como objetivo avaliar os determinantes epidemiológicos do Traumatismo Cranioencefálico (TCE) no paciente pediátrico. Foi realizada uma revisão integrativa através da busca de artigos indexados na Biblioteca Virtual em Saúde, no qual a análise após analise final foram incluídos 08 artigos. Para melhor elucidação foram formuladas duas categorias: Perfil epidemiológico de crianças com TCE e Determinantes epidemiológicas e as implicações clinicas do TCE em crianças. Foi possível verificar que existe uma predominância do sexo masculino, a queda é considerada mecanismo principal para o quadro e TCE, além disso, faixas etárias inferiores a 5 anos apresentam pior prognóstico.
\end{abstract}

Descritores: Traumatismos craniocerebrais; Criança; Fatores Epidemiológicos.

\section{Introdução}

O trauma na região da cabeça é considerado como um dos principais fatores de óbito e incapacidade em todo o globo, apresentando mundialmente uma incidência anual em torno de 200 internações a cada 100000 habitantes anualmente. No qual é notório que existe uma distribuição trimodal quando se avalia a faixa etária das vitimas, apontando picos principalmente na primeira infância, adolescência tardia e indivíduos com idade superior 60 anos. Quando se analisa a presença de lesões na cabeça na faixa etária infantil é identificada como um agravo com prejuízos no âmbito da saúde pública no qual esta associada principalmente a um elevado potencial de prejuízos ao longo da vida (Gabbe et al.,2014).

Guerra e outros colaboradores (2010) descrevem que o trauma promove anualmente a morte de 22 mil crianças e adolescentes por ano no país, sendo que $85 \%$ dos casos graves apresentam traumatismo cranioencefálico (TCE), sendo o principal fator para a taxa de mortalidade, além disso, acarreta diversas sequelas aos sobreviventes. Os danos secundários que ocorre no encéfalo são considerados os principais fatores relacionados a um pior prognóstico, entre eles destaca-se hipoxemia, hipotensão e hipertensão intracraniana (HIC). Diante desse contexto, durante a abordagem terapêutica deve-se lançar mão de alternativas que proporcione corrigir ou evitar a formação desses 
fatores, principalmente o controle da pressão intracraniana (PIC) e a manutenção da pressão de perfusão encefálica (PPE) e com isso garantido uma adequada oferta de oxigênio ao encéfalo.

O TCE não acidental está associado a diversas implicações clínicas e psicossociais inevitáveis, principalmente na faixa etária infantil no qual é notório que afeta de maneira significativa o desenvolvimento neuropsicomotor, como também esta associada a uma elevada morbimortalidade (Matsubara et al.,2018).

Santiago e outros colaboradores (2018) afirmam que o traumatismo cranioencefálico decorrente em pacientes meninil possui diversas características clínicas que apresentam influência sobre o diagnóstico e gravidade do agravo. Diante disso, são características importantes a idade da vítima, a escala de coma Glasgow, o aparecimento ou ausência de alteração pupilares, como também, a classificação da tomografia computadorizada que mede a gravidade da lesão. Esse arquétipo de trauma é um dano cerebral que o paciente adquiri devido algum incidente de algum tipo de choque ou golpe na região da cabeça, resultando em uma disfunção cerebral anormal e ocorrendo a possibilidade de gerar ou não sintomas a depender do local lesionado, da adição dos danos causados e sobretudo da faixa etária da criança.

Diante desse contexto, pode-se afirmar que o quadro de TCE na faixa etária infantil é considerada um grande problema de saúde pública, no qual torna-se fundamental proporcionar ferramentas de prevenção. Para facilitar a construção dessas estratégias é fundamental identificar os determinantes epidemiológicos de crianças com TCE, como também suas implicações clinicas. Portanto, este trabalho tem com objetivo avaliar os determinantes epidemiológicos do TCE no paciente pediátrico.

\section{Métodos}

Foi realizada uma revisão integrativa, com abordagem descritiva e exploratória, no qual o primeiro passo foi a definição maneira precisa o problema da pesquisa, com posterior escolha das bases de dados e busca na literatura; categorização dos estudos; avaliação dos estudos incluídos na revisão; extração dos principais resultados, organização e análise dos dados obtidos (Mendes; Silveira; Galvão, 2008). 
Desta forma, o objeto de estudo deste trabalho foi a produção científica sobre o tema, existente em periódicos indexados. Para a realização da busca, foram utilizadas combinações entre as seguintes descritoras no DeCS (Descritores em Ciências de Saúde): “Traumatismos craniocerebrais" AND “Criança” AND "Fatores Epidemiológicos".

Os termos foram cruzados como descritores e também como palavras do título e do resumo. A busca foi realizada no período de dezembro de 2019, através do cruzamento dos descritores de dois a dois com o objetivo de identificar o maior número de evidências possível, como conectivo foi utilizado o operador boleano "AND”. A pesquisa foi norteada a partir da seguinte questão: Quais os determinantes epidemiológicos do TCE no paciente pediátrico?

Como critérios de inclusão, foram incluídas as publicações: (a) ocorridas entre 2009 e 2019; (b) em qualquer idioma (c) que abordaram o perfil epidemiológico de pacientes pediátricos com TCE d) artigos com texto completo disponível online e) Artigos que analisassem as implicações clinicas do TCE na faixa etária infantil; excluíram-se os artigos que: (a) não abordaram o perfil epidemiológico dos pacientes; (b) não localizados na íntegra; (c) dissertações, teses e cartas ao editor.

A busca de artigos foi realizada por meio da Biblioteca Virtual em Saúde (BVS). Os estudos potencialmente relevantes para serem incluídos na revisão integrativa foram obtidos pela leitura dos títulos, resumos e do texto integral sempre que o resumo não fosse esclarecedor relativamente aos critérios utilizados para a decisão de inclusão ou exclusão.

\section{Resultados e discussões}

$\mathrm{Na}$ fase de identificação dos artigos, obtiveram-se 22 artigos, dos quais 06 estavam localizados na Literatura Latino-americana e do Caribe em Ciências da Saúde (LILACS), 15 no Sistema Online de Busca e Análise de Literatura Médica (MEDLINE) e 1 no Índice Bibliográfico Espanhol de Ciências de Saúde (IBECS). Posteriormente 12 foram excluídos após a análise dos critérios de inclusão, por serem inconclusivos, com baixo teor metodológico. Através da leitura detalhada dos estudos e pela confirmação de elegibilidade, 8 artigos foram inclusos como evidências por responderem aos objetivos do estudo. 
O tipo do estudo foi retrospectivo em três estudos, prospectivo em dois e três foram estudo de coorte. Dos 08 estudos analisados, quanto às características gerais, a publicação mais antiga era de 2010; seis (75\%) artigos eram nacionais e dois (25\%) tinham abrangência internacional. Foi então produzida a Tabela 1 no qual descreve as características dos estudos selecionados e seu desfecho. A Figura 1 demonstra o Fluxograma da busca dos estudos da revisão integrativa.

Fluxograma 1- Estratégia de busca com os descritores: "Traumatismos craniocerebrais" AND “Criança” AND "Fatores Epidemiológicos", Juazeiro do Norte- CE, 2019

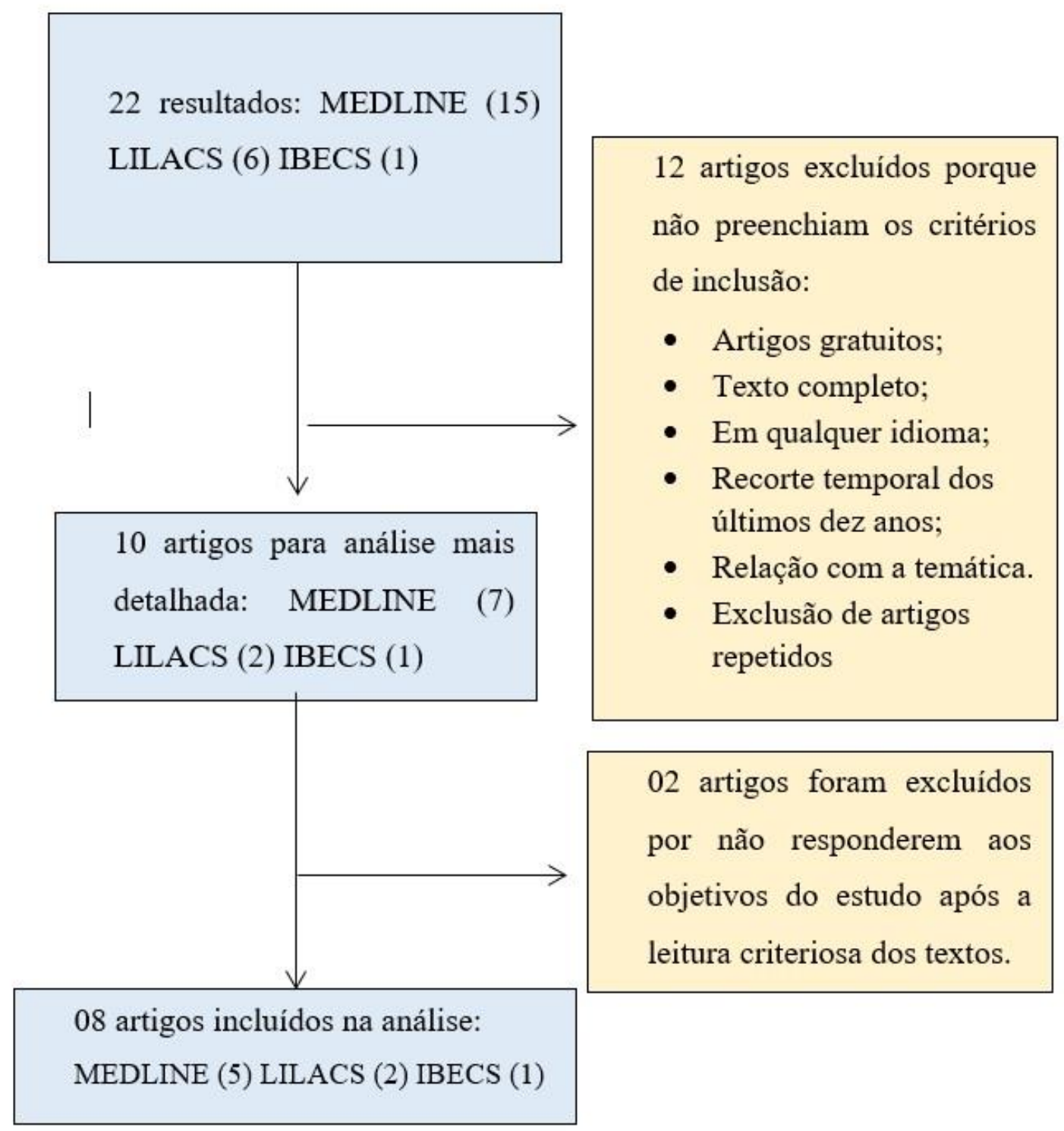

Fonte: Elaborado pelos autores. 
Durante a análise da Tabela 01 é possível identificar que o principal trauma nas crianças é o TCE, em que a mecanismo do trauma depende da faixa etária, no qual os mais comuns são queda, acidentes de trânsito e violência. Além disso, é notória uma predominância do sexo masculino na maior dos estudos.

Tabela 01: Características dos estudos selecionados, Juazeiro do Norte-CE, 2019.

\begin{tabular}{|c|c|c|c|c|c|}
\hline $\begin{array}{c}\text { Autor/ } \\
\text { Ano }\end{array}$ & País & Periódico & Titulo & $\begin{array}{l}\text { Tipo de } \\
\text { Estudo }\end{array}$ & Desfecho \\
\hline $\begin{array}{l}\text { Aguiar, } \\
2013\end{array}$ & Brasil & Cirurgiões & $\begin{array}{l}\text { Traumatismo } \\
\text { cranioencefálico por } \\
\text { projétil de arma de } \\
\text { fogo: experiência de } 16 \\
\text { anos do serviço de } \\
\text { neurocirurgia da Santa } \\
\text { Casa de São Paulo. }\end{array}$ & Prospectivo & $\begin{array}{l}\text { Foi realizada a avaliação } \\
\text { epidemiológica de pacientes } \\
\text { com TCE decorrente de PAF } \\
\text { no qual foi possível } \\
\text { identificar um predomínio do } \\
\text { sexo masculino, com lesão } \\
\text { principalmente do lobo } \\
\text { frontal. Além disso, os } \\
\text { autores afirmam que } \\
\text { abordagem cirúrgica é } \\
\text { fundamental para } \\
\text { proporcionar um melhor } \\
\text { prognóstico, especialmente } \\
\text { em pacientes mais graves. }\end{array}$ \\
\hline $\begin{array}{l}\text { Amori } \\
\text { m et al., } \\
2017\end{array}$ & Brasil & $\begin{array}{l}\text { Rev. enferm. } \\
\text { UFPE on line }\end{array}$ & $\begin{array}{l}\text { Perfil epidemiológico } \\
\text { de crianças vítimas de } \\
\text { trauma } \\
\text { cranioencefálico }\end{array}$ & Estudo de coorte & $\begin{array}{l}\text { Ao avaliar o perfil de } \\
\text { crianças com TCE foi } \\
\text { identificada uma } \\
\text { predominância do sexo } \\
\text { masculino e com idade } \\
\text { inferior a } 2 \text { anos, sendo a } \\
\text { queda o principal fator casual } \\
\text { e o TCE leve o mais } \\
\text { identificado. }\end{array}$ \\
\hline $\begin{array}{l}\text { De } \\
\text { Fatima } \\
\text { Simas; } \\
\text { Da } \\
\text { Silva } \\
\text { Souza, } \\
2019\end{array}$ & Brasil & $\begin{array}{l}\text { Revista Pró- } \\
\text { UniverSUS }\end{array}$ & $\begin{array}{l}\text { Perfil de crianças } \\
\text { hospitalizadas na } \\
\text { pediatria vítimas de } \\
\text { acidentes na primeira } \\
\text { infância }\end{array}$ & Retrospectivo & $\begin{array}{l}\text { Ao avaliar o perfil de } \\
\text { pacientes pediátricos de } 0 \text { a } 6 \\
\text { anos internado decorrentes de } \\
\text { acidentes, foi possível } \\
\text { identificar a queda como } \\
\text { principal mecanismo gerador } \\
\text { do TCE, acometendo } \\
\text { principalmente crianças do } \\
\text { sexo masculino. }\end{array}$ \\
\hline $\begin{array}{l}\text { Gabbe } \\
\text { et al., } \\
2014\end{array}$ & $\begin{array}{l}\text { País de } \\
\text { Gales }\end{array}$ & $\begin{array}{l}\text { Epidemiol } \\
\text { Community } \\
\text { Health }\end{array}$ & $\begin{array}{l}\text { The association } \\
\text { between hospitalisation } \\
\text { for childhood head } \\
\text { injury and academic } \\
\text { performance: evidence } \\
\text { from a population e- } \\
\text { cohort study }\end{array}$ & Estudo de Coorte & $\begin{array}{l}\text { O estudo em questão avaliou } \\
\text { os prejuízos no desempenho } \\
\text { acadêmica de crianças com } \\
\text { lesões na cabeça, no qual foi } \\
\text { identificado que o TCE esta } \\
\text { associado a implicações } \\
\text { importantes do } \\
\text { desenvolvimento cognitivo } \\
\text { dos pacientes. }\end{array}$ \\
\hline
\end{tabular}




\begin{tabular}{|c|c|c|c|c|c|}
\hline $\begin{array}{l}\text { Guerra } \\
\text { et al., } \\
2010\end{array}$ & Brasil & $\begin{array}{l}\text { Jornal de } \\
\text { pediatria }\end{array}$ & $\begin{array}{l}\text { Factors associated with } \\
\text { intracranial } \\
\text { hypertension in } \\
\text { children and teenagers } \\
\text { who suffered severe } \\
\text { head injuries. }\end{array}$ & Estudo de Coorte & $\begin{array}{l}\text { Foi realizada a avaliação de } \\
\text { crianças com TCE e suas } \\
\text { implicações clinicas, no qual } \\
\text { tornou-se notório que o } \\
\text { quadro de HIC e HIC } \\
\text { refratárias são os principais } \\
\text { fatores de morbimortalidade, } \\
\text { principalmente em crianças } \\
\text { com faixa etária menor. }\end{array}$ \\
\hline $\begin{array}{l}\text { Matsub } \\
\text { ara et } \\
\text { al., } \\
2018\end{array}$ & Brasil & $\begin{array}{l}\text { Arquivos } \\
\text { Brasileiros de } \\
\text { Neurocirurgia: } \\
\text { Brazilian } \\
\text { Neurosurgery }\end{array}$ & $\begin{array}{l}\text { Fatores de prognóstico } \\
\text { em crianças menores } \\
\text { de } 5 \text { anos vítimas de } \\
\text { trauma } \\
\text { cranioencefálico não } \\
\text { acidental em Curitiba }\end{array}$ & Retrospectivo & $\begin{array}{l}\text { Ao avaliar o prognóstico de } \\
\text { crianças menores de } 5 \text { anos } \\
\text { com TCE é notório a } \\
\text { identificação de uma } \\
\text { gravidade associada a } \\
\text { múltiplas lesões, gerando a } \\
\text { necessidade de um } \\
\text { atendimento ágil e de } \\
\text { qualidade. Além disso, foi } \\
\text { observado uma taxa de } \\
\text { mortalidade de } 12 \% \text { em que } \\
40 \% \text { dos pacientes foram } \\
\text { submetidos à intervenção } \\
\text { cirúrgicas. }\end{array}$ \\
\hline $\begin{array}{l}\text { Pachec } \\
\text { o et al., } \\
2014\end{array}$ & Espanha & $\begin{array}{l}\text { Anales de } \\
\text { Pediatría }\end{array}$ & $\begin{array}{l}\text { Accidentes de bicicleta } \\
\text { atendidos en los } \\
\text { Servicios de Urgencias. } \\
\text { Estudio multicéntrico. }\end{array}$ & Prospectivo & $\begin{array}{l}\text { Realizado um estudo com } \\
\text { objetivo de avaliar os fatores } \\
\text { epidemiológicos de pacientes } \\
\text { atendidos após acidente de } \\
\text { bicicleta, no qual identificou } \\
\text { que o TCE proporcionou a } \\
\text { admissão em } 68,4 \% \text { dos } \\
\text { casos. }\end{array}$ \\
\hline $\begin{array}{l}\text { Santiag } \\
\text { o et al., } \\
2018\end{array}$ & Brasil & $\begin{array}{l}\text { Anais do } \\
\text { Seminário } \\
\text { Científico da } \\
\text { FACIG }\end{array}$ & $\begin{array}{l}\text { Perfil epidemiológico } \\
\text { do traumatismo } \\
\text { cranioencefálico } \\
\text { pediátrico }\end{array}$ & Retrospectivo & $\begin{array}{l}\text { Estudo retrospectivo em que } \\
\text { descreveu a maior parte dos } \\
\text { traumas na faixa etária } \\
\text { infantil ocorre em domicilio, } \\
\text { e o TCE é considerado o } \\
\text { principal mecanismo de } \\
\text { morte. }\end{array}$ \\
\hline
\end{tabular}

Fonte: Elaborado pelos próprios autores

Para melhor elucidação da discussão, as categorias consideradas relevantes foram categorizadas da seguinte forma:

Perfil epidemiológico de crianças com TCE

Pacientes com TCE está associado a diversas alterações clínicas que promovem uma elevada taxa de morbimortalidade, com complicações e sequelas que prejudicam a vida do 
paciente, principalmente em crianças. Durante a análise é possível verificar que o sexo predominante foi principalmente o masculino, especialmente quando o trauma foi em crianças com idades menores, sendo a queda nesse grupo o principal mecanismo de trauma, gerando assim, principalmente um TCE leve. Vale salientar que à medida que a criança vai ficando mais ocorrem modificações nesse perfil.

A Organização Mundial da Saúde (OMS) afirma que o TCE como qualquer tipo de trauma que gere lesão anatômica ou comprometimento funcional do couro cabeludo, crânio, meninges ou encéfalo, no qual ele é classificado clinicamente em três tipos: traumatismo craniano fechado, fraturas com afundamento de crânio e fratura exposta do crânio. Além disso, através da análise da Escala de Glasgow, sintomatologia e resultados identificados pelos exames de imagem ele é classificado pela gravidade, em leve, moderado e grave (Amorim et al.,2017).

Corroborando, Santiago e outros colaboradores (2018) descrevem que a lesão em pacientes pediátricos há uma dessemelhança desta em adultos, visto que o cérebro infantil está em processo de desenvolvimento havendo a possibilidade dos sintomas tardarem no aparecimento. Além do mais, nos pacientes infantis o trauma é um processo de doença crônica ao adverso dos adultos que se manifesta rapidamente e de uma só vez. O TCE pode ser entendido de acordo com a gravidade sendo de forma leve, moderado ou grave, tendo em vista o tamanho e natureza da lesão, com a quantidade de tempo de perca da consciência com a perda da memória pós-traumática e com a gravidade da conclusão durante o exame realizado.

O TCE em crianças e adolescentes é caracterizado por promover alterações no âmbito físico e psíquico, no qual é considerado como um dos principais tipos de trauma infantil, apresentando taxa de morbidade e mortalidade que se aproxima de $75 \%$ e $97 \%$ respectivamente (Amorim et al.,2017).

Um estudo retrospectivo com 182 pacientes com TCE decorrentes de projetil de arma de fogo (PAF) ao avaliar o perfil epidemiológico foi possível identificar que $22 \%$ dos pacientes estavam na faixa etária entre 11 a 20 anos, no qual a maior parte era do sexo masculino e em $84 \%$ das situações ocorreu penetração da dura máter, que esta associada a um pior prognóstico (Aguiar et al.,2013).

Amorim e outros colaboradores (2017) realizaram um estudo de coorte no qual analisaram 142 prontuários de crianças de 01 a 04 anos vitimas de TCE, em que foi 
possível identificada que $62,67 \%$ dos pacientes eram do sexo masculino, $64,08 \%$ com idade entre 01 e 02 anos, sendo a maior parte dos casos considerada um TCE leve.

Um estudo de coorte avaliou 290 crianças com idade entre 5 a 7 anos que foram atendidas decorrente de lesões na cabeça, no qual foi possível verificar que $52,7 \%$ dos pacientes apresentaram fratura craniana e em 36,8\% das admissões foram por lesões intracranianas, no qual existiu uma predominância de crianças com idade inferior a 3 anos, sendo mais comum no sexo masculino(Gabbe et al.,2014).

Guerra e outros colaboradores (2010) realizaram um estudo de coorte no qual avaliaram 132 crianças que receberam monitorização da PIC decorrente de um quadro de TCE, no qual foi possível identificar um predomínio de pacientes do sexo masculino, com faixa etária entre 7 e 9 anos, sendo os acidentes de trânsito o principal mecanismo de trauma, além disso a maior parte dos pacientes apresentavam múltiplas lesões intracranianas.

Matsubara e outros colaboradores (2018) avaliaram o perfil epidemiológico de 24 crianças com faixa etária inferior a 5 anos com quadro de TCE não acidental, no qual constatou-se que a queda da própria altura como fator causal, representando $70 \%$ dos casos, acometendo principalmente a faixa etária inferior a 3 anos e do sexo feminino. Nesse contexto, é fundamental o estabelecimento de medidas de prevenção de quedas, para que se possa proporcionar a redução desse agravo. Além disso, a violência infantil é fator identificado como mecanismo de trauma, apresentando uma taxa de $3 \%$.

Um estudo multicêntrico e prospectivo com 846 pacientes de 3 a 16 anos atendidos após acidente de bicicleta foi possível identificar o TCE como principal causa de admissão, sendo que $77,9 \%$ não faziam uso de capacete e $72,9 \%$ eram do sexo masculino. Diante desse processo chama à atenção a necessidade de promover estratégias com foco no uso de aparelhos se segurança, já que o capacete é considerado a principal ferramenta de proteção, já que promove a diminuição de lesões do sistema nervoso central devido o TCE em $88 \%$ dos casos (Pacheco et al., 2014).

Um estudo retrospectivo revelou que crianças arremetidas pelo traumatismo cranioencefálico, com idade abaixo de 10 anos, ultrapassam de $20 \%$ das internações hospitalares entre a faixa de 0 a 4 anos. Da mesma forma estudos epidêmicos realçam que pacientes do sexo masculino têm um maior número de chances de sofrer o TCE em relação ao sexo feminino, e também como supracitado são crianças de 0 a 4 anos possuindo maior chance de ter esse trauma comparado aos outros. Tudo isso deve-se ao fato de que maior 
parte dos meninos estão exibidos a fatores arriscados, como brincadeiras arriscadas que podem levar a quedas consideráveis. Vale ressaltar também que as crianças ainda não possuem a capacidade de julgar completamente os fatores disponíveis a acidentes. De maneira igualitária, é imprescindível dizer que o ambiente doméstico é um dos locais de acidente em relação a existência de possíveis " Situações Perigosas" como a facilidade a materiais perfuro-cortante ou mesmo o local favorece, por meio de escadas e pisos escorregadios ou pelo baque da própria altura, levando assim a um TCE. Vale frisar que as quedas são os principais motivos do TCE em crianças com faixa etária abaixo de 5 anos (Santiago et al.,2018).

\section{Determinantes epidemiológicas e as implicações clínicas do TCE em crianças}

O quadro de TCE na faixa etária é acompanhando com uma elevada taxa de mortalidade, no qual gera diversas implicações clínicas no qual é essencial o estímulo de estratégias de prevenção. De maneira geral promove alterações no âmbito social, educacional, econômico e afeta de maneira significativa na qualidade de vida das crianças, sendo o controle das complicações e monitorização do paciente, ferramenta chave para diminuição da morbidade, além disso, foi possível identificar a adoção de medidas de prevenção é crucial. Outro ponto é que apesar de apresentar uma etiologia multifatorial, os principais mecanismo são as quedas e os acidentes.

Aguiar (2013) descreve que pacientes com TCE decorrentes de projetil de arma de fogo (PAF) existem um impacto significativo no contexto social e econômico, já que a maior parte dos pacientes estão dentro da faixa etária economicamente ativa, além disso, devido a energia gerada por este tipo de trauma pode gerar lesões ósseas e nervosas, como os lobos e com isso esse processo esta associado a uma elevada morbimortalidade, sendo assim, um grande desafio terapêutico. Diante desse contexto a realização de procedimentos cirúrgicos é considerada ferramenta crucial para proporcionar uma menor taxa de mortalidade.

A queda representou $95,65 \%$ como mecanismo de trauma, principalmente em crianças com idade inferior a 3 anos enquanto que, 6,45\% foram decorrentes de acidentes de trânsito. Nesse processo, a adoção de medidas de promoção da saúde são fundamentais para traçar alternativas de prevenção desse agravo, já que apresenta uma elevada morbimortalidade. Medidas como acompanhamento, a supervisão adequada de crianças, 
uso da cadeirinha e do cinto se segurança são exemplos de ferramentas cruciais para diminuição da incidência de TCE (Amorim et al.,2017).

Corroborando com o fato supracitado, De Fátima Simas e Da Silva Souza (2019) estabelecem que os acidentes que acometem a faixa etária infantil nos últimos anos sua taxa cresceu consideravelmente, com isso se tornando um grave problema de Saúde Pública, podendo esta associada a diversos prejuízos, entre eles incapacidade física temporária, sequelas e óbito. De acordo com dados Organização Mundial de Saúde, anualmente decorrente de acidentes o número de mortes ultrapassa 5 milhões. Neste contexto, em um estudo retrospectivo com 50 crianças atendidas num setor de internação pediátrica, avaliou os principais acidentes na primeira infância, no qual foi possível identificar que $68 \%$ dos casos admitidos foram decorrentes de quedas e apresentaram como diagnóstico o TCE. Além disso, os autores descrevem que as outras causas que promovem TCE na faixa etária infantil, são principalmente acidentes de trânsito e acidentes com bicicleta.

Diante desse panorama, vale salientar que é fundamental a realização de um atendimento de qualidade e integral, no qual a análise de todos os componentes é fundamental para controle e introdução de medidas que proporcione uma melhor qualidade de vida. A atenção a sinais de gravidade são fatores essenciais, como por exemplo, o controle de vômitos, a identificação de hipertermia. No estudo em questão 35,9\% dos pacientes apresentaram algum tipo de complicação, sendo que os principais foram a formação de hematoma subgaleal, trauma raquimedular, parada cardiorrespiratória, coleção subdural, hematoma gabal, Hematoma Extradural Agudo (HEDA) e infecção de face, principalmente a celulite. Por fim, foi identificada uma taxa de mortalidade de $0,7 \%$, dado este decorrente da maior parte dos casos terem sido um TCE leve, além disso, difere da maioria dos estudos recentes que descrevem uma taxa superior a 20\% (Amorim et al., 2017).

As lesões que ocorrem na cabeça em crianças, decorrentes de traumatismos cranianos, principalmente com trauma intracraniano, apresenta um resultado crítico associado a prejuízos cognitivos, emocionais, funcionais e comportamentais. Os estudos recentes descrevem uma dificuldade de atingir desempenhos acadêmicos adequados após sofrer um TCE, em que a gravidade da lesão esta associada a fatores de comportamento e déficits na memória. Outras alterações que podem ocorrer são comprometimento da habilidade motora e sequelas neurocognitivas. Neste contexto, é crucial a introdução de 
medidas que busquem a diminuição de lesões na cabeça na faixa etária infantil, como também o estabelecimento de estratégias de reabilitação escolar (Gabbe et al.,2014).

Durante a avaliação de 132 crianças com TCE identificou-se que aproximadamente 80\% dos pacientes apresentaram HIC, principalmente em crianças com faixa etária inferior a 4 anos, no qual a monitorização da PIC é fundamental para proporcionar um melhor

controle. É importante compreender que o controle adequado é fundamental, especialmente nos casos de HIC refratária no qual esta associada a uma elevada morbimortalidade. A alternativa mais viável para a monitorização desses pacientes é com o parafuso de Richmond, além disso a avaliação clinica e radiográfica são ferramentas cruciais quando a primeira opção não esta disponível ou ocorre falha (Guerra et al., 2010).

Matsubara e outros colaboradores(2018) descrevem que o TCE em crianças com faixa etária inferior a 5 anos esta associado a um mal prognóstico, principalmente decorrente do resultado das lesões, em que 70\% dos apresentam afundamento de crânio, fraturas e múltiplas lesões cranianas e com isso gerando um TCE grave em que um atendimento adequado, eficaz, monitorização e intervenção cirúrgica são consideradas estratégias chaves na abordagem do paciente, já que a taxa de óbito é superior a $10 \%$.

\section{Considerações finais}

Diante da análise dos estudos é possível afirmar que o sexo masculino apresenta predominância de acometimento de TCE na faixa etária infantil, sendo a queda o principal mecanismo. Além disso, existem diversas implicações que envolve desde o âmbito social ao econômico, em que se destaca, que quando menor a faixa etária pior o prognóstico do paciente. Sendo assim, crucial o estabelecimento de medidas adequadas que promovam um melhor atendimento do paciente, focando na monitorização e avaliando a necessidade de procedimentos cirúrgicos.

Vale salientar que existe uma elevada morbimortalidade associada ao quadro de TCE na infância, sendo assim crucial o estabelecimento de estratégias em promoção em saúde com o objetivo de diminuir os fatores casuísticos. Diante disso, é fundamental a análise dos determinantes epidemiológicos e com isso torna-se essencial a realização de mais estudos com foco nessa temática, já que é notório que existem poucos estudos na área. 


\section{Referências}

Aguiar, D. E. (2013). Traumatismo cranioencefálico por projétil de arma de fogo: experiência de 16 anos do serviço de neurocirurgia da Santa Casa de São Paulo. Cirurgiões, v. 40, n. 4, p. 300-304.

Amorim, Elizabeth de Souza et al. (2017). Perfil epidemiológico de crianças vítimas de trauma cranioencefálico. Rev. enferm. UFPE on line, v. 11, n. supl. 10, p. 4150-4156.

De Fatima Simas, Vanessa; Da Silva Souza, Alessandra (2019). Perfil de crianças hospitalizadas na pediatria vítimas de acidentes na primeira infância. Revista PróUniverSUS, v. 10, n. 1, p. 25-28.

Gabbe, Belinda J. et al. (2014). The association between hospitalisation for childhood head injury and academic performance: evidence from a population e-cohort study. $J$ Epidemiol Community Health, v. 68, n. 5, p. 466-470.

Guerra, Sérgio Diniz et al. (2010). Factors associated with intracranial hypertension in children and teenagers who suffered severe head injuries. Jornal de pediatria, v. 86, n. 1, p. 73-79.

Matsubara, Anderson et al. (2018). Fatores de prognóstico em crianças menores de 5 anos vítimas de trauma cranioencefálico não acidental em Curitiba. Arquivos Brasileiros de Neurocirurgia: Brazilian Neurosurgery, v. 37, n. S 01, p. A1352.

Mendes, K. D. S.; Silveira, R. C. C. P.; Galvão, C. M. (2008). Revisão integrativa: método de pesquisa para a incorporação de evidências na saúde e na enfermagem. Texto \& Contexto Enfermagem. Florianópolis, v. 17, n. 4, p.758-764, out/dez.

Pacheco, N. González et al. (2014). Accidentes de bicicleta atendidos en los Servicios de Urgencias. Estudio multicéntrico. In: Anales de Pediatría. Elsevier Doyma, v. 80, n. 4, p. 242-248.

Santiago, Luíza Gomes et al. (2018). Perfil Epidemiológicodo Traumatismo Cranioencefálico Pediátrico. Anais do Seminário Científico da FACIG, n. 3.

\section{How to cite this article (APA format):}

Gonçalves, Daniel Victor Lima; Gonçalves; Leite, Pedro de Sousa; Mgalhães, Bráulio Filgueira; Cruz, Emanuela Fonseca; Cruz, Amanda Fonseca; Balduino, Cicero Hyttallo Carneiro; Saraiva, Emanuelly Gonçalves; Alencar, Thiago Moreira de; Feitosa, Natália Moreira Garcia; Feitosa, Virgínia Moreira Garcia. (2019). Epidemiological Determinants of Cranioencephalic Trauma in Children. Am. In. Mult. J., October a December. (7) 4, 232-244.

Received: $11 / 25 / 2019$

Accepted: 12/12/2019 